\title{
Student-identified themes around computation in high school physics
}

\author{
Theodore E. Bott and Daniel P. Weller \\ Department of Physics \& Astronomy, Michigan State University, 567 Wilson Rd., East Lansing, MI, 48824 \\ Marcos D. Caballero \\ Department of Physics \& Astronomy, Michigan State University, 567 Wilson Rd., East Lansing, MI, 48824 \\ CREATE for STEM Institute, Michigan State University, 567 Wilson Rd., East Lansing, MI, 48824 and \\ Department of Physics \& Center for Computing in Science Education, University of Oslo, N-0316 Oslo, Norway \\ Paul W. Irving \\ Department of Physics \& Astronomy, Michigan State University, 567 Wilson Rd., East Lansing, MI, 48824
}

In response to the growing emphasis on computational thinking in recent multi-state education standards, as well as the ever-changing applicability of computation in both educational and professional realms, we are developing a survey which evaluates the attitudes of students exposed to computational learning activities in their high school physics classrooms. We administered a pilot questionnaire in classrooms with computation integrated into their physics curricula and conducted a thematic analysis on student responses. Several expected themes (identified previously from teacher interviews) and emergent themes (emerging from open-ended responses to the survey) arose in our analysis. This work focuses on three main themes: the applicability of physics ideas in coding, computational thinking practices, and the usefulness of computation. We provide an in-depth discussion of response variation and its impact on future survey development. This work lays the foundation for the development of a robust and validated survey that assesses students' opinions, expectations, and attitudes towards learning computation in their science classes.

2019 PERC Proceedings edited by Cao, Wolf, and Bennett; Peer-reviewed, doi.org/10.1119/perc.2019.pr.Bott Published by the American Association of Physics Teachers under a Creative Commons Attribution 4.0 license. Further distribution must maintain attribution to the article's authors, cover page, and DOI. 


\section{INTRODUCTION}

There are multitudinous justifications for integrating computation into high school science classrooms. First, a plethora of modern science professions demand a basic understanding of coding. Additionally, the Next Generation Science Standards (NGSS) emphasize a development of computational thinking practices as early as kindergarten, with more involved standards required up to twelfth grade [1]. Beyond high school, computation has grown into a focal point of many introductory physics, engineering, and math courses at the collegiate level [2]. Therefore, the inclusion of computational thinking practices in science education standards, compounded with the ubiquity of computation in the professional realm, motivated our investigation of students' attitudes toward the topic in high school physics.

Several prominent works in the field of physics education, specifically epistemological survey creation, informed the development of this project: the Colorado Learning Attitudes about Science Survey (CLASS) [3], its experimental-specific counterpart E-CLASS [4], the Views About Science Survey (VASS) [5], and Maryland Physics Expectations (MPEX) survey [6]. Of these, we chose to center our process around CLASS and E-CLASS. Whereas CLASS lays the foundation for a typical affect-based survey to broadly examine physics students, E-CLASS provides a definitive example of modifying the CLASS survey for a specific context (a physics lab, as opposed to a physics classroom). In accordance with E-CLASS, we followed a similar process of iterative validation and refinement in designing our questionnaire, only with regard to computation as opposed to laboratory content.

The overall goal of the work presented here is to identify major themes from student responses that will lay the groundwork for the creation of a closed-form, statement-based survey. Analysis of these themes is the preliminary stage in developing a large-scale, multifaceted survey that will be adaptable to teachers' specific intended learning outcomes around computation. This survey should allow for relatively straightforward analysis, coincide with existing scholarly literature that evaluates student affect, and incorporate the themes revealed by students in this early stage of development. These student-identified themes will ultimately serve as the basis for statement design and structure in the final survey. We hope to establish a survey that allows teachers to easily and effectively evaluate their students' attitudes towards computational activities and themselves as instructors.

\section{QUESTIONNAIRE DESIGN AND ANALYSIS}

Before devising attitudinal questions, we conducted three interviews with high school physics teachers to illuminate their learning goals around computation. Then, four questions were chosen from CLASS that most aligned with the teachers' objectives, and the question language was reshaped to make them computation-focused rather than physics- focused. CLASS served as a basis for our framework because it covered a broad range of topics and comprised questions that were easily altered to be asked through a computational lens. Framing CLASS questions in the context of computation was often as straightforward as changing the word "physics" to "computation" or "coding." However, this process also included computation-specific terms that were not in the original wording of the prompt, such as "understanding every line of code" (in question 4 displayed below). These questions were reviewed by other researchers to assure that they were aligned with our research goals.

The preliminary four-question, open-ended pilot questionnaire was given to a class of 15 students. Initial analysis revealed that a significant portion of the responses were strictly binary (i.e., students responded "yes" and "no," or "important" and "not important" to questions that were intended to be free response). Thus, we added an open-ended question at the beginning to elicit students' self-identified ideas and get them comfortable with the questionnaire before moving on to more complex inquiries. We also included a second question to explore the idea of usefulness that emerged from initial analysis. To avoid binary responses, wording was edited to ask questions through a larger scope (i.e., words like "how" replaced "do", and phrases like "please explain" were added to induce more thorough answers). In this sense, the questionnaire was designed to be exploratory and provide a large pool of varied responses. The initial questions are documented here: [11]. Listed below are the final six questions:

1. How do you feel about learning computation in your science class? Please explain.

2. Why is computation useful for science? Please explain.

3. Based on your experience, how easy/difficult has it been to learn coding in your science class? Please explain.

4. When coding, do you think it is important to understand every line of the code before you can use it correctly? Why or why not? Please explain.

5. When using code to solve a problem, how do your physics ideas apply to the problem? Please explain.

6. How does learning coding change your ideas about how physics works? Please explain.

For our official implementation, we administered the questionnaire to $N=61$ students in grades $10-12$ from three separate classes ( 1 physics and 2 physical science) under one teacher at a private school. Participants filled out the questionnaire after multiple experiences with computation (GlowScript, VPython) to evoke more comprehensive responses. We then conducted a thematic-analysis, which employed a mix of a priori (deductive) and generative (inductive) elements, to investigate expected themes identified from teacher interviews and any new ideas that emerged.

Our thematic analysis approach was heavily informed by the framework outlined by Otero and Harlow, which consists of 5 steps for analyzing participants' responses: (1) read transcripts, noting initial ideas; (2) pick one transcript...and write 
TABLE I. Themes in students responses relating to applicability (APP) of physics ideas when coding. ${ }^{\text {a }}$

\begin{tabular}{ll}
\hline \hline Sub-themes & Example Response \\
\hline PS - Physics-specific (30/61) & "Newtons laws apply, along with things like speed, velocity, acceleration, time, etc." \\
EQ - Equations (7/61) & "Physics is a lot of equations and plugging in numbers, and that is very similar to coding." \\
L - Learnability (9/61) & "Knowing the physics behind the coding can help put the code together and understand what you are doing." \\
ID - Interdisciplinary (14/61) & "They are the basis of all the code, so it is necessary to understand the physics basics." \\
O - Other (14/61) & Other section refers to unspecified agreements, such as "Yes my physics ideas apply." \\
\hline \hline
\end{tabular}

a Numbers in parentheses represent the count of students that mentioned each sub-theme out of the total set of students surveyed.

meanings in 2-3 word phrases; (3) begin coding by identifying segments of text that relate to a particular code; (4) make a list of all the codes and group them together; (5) go back to the data and try to code using this scheme, refining or removing codes whenever necessary [9]. Our analysis process was completed at two grain sizes. The initial grain size comprised an examination of responses as a whole body of data to explore themes related to student ideas and perceptions that overlapped between questions. This initial analysis resulted in no crosscutting themes being identified beyond themes constituted within the responses of individual questions. In the end, five high-level themes were identified: applicability of physics ideas when coding, computational thinking practices, usefulness of computation, importance of understanding every line of code, and difficulty learning computation.

Our second level of analysis aimed at investigating themes within the aforementioned higher level themes. Repeating the process outlined above, we read through all the responses within a theme holistically to a get a general sense for the ideas presented. For each response, we wrote out what our interpretation of the response was, making sure to use text from the student response as justification for that interpretation. These interpretations were then coded and grouped together to create sub-themes. The sub-themes were used to code the data within a higher level theme, again making sure to refine sub-themes when necessary. Within each higher level theme, multiple sub-themes emerged which will be described as the variation within a higher level theme. After coding the responses on these two levels, we conducted an inter-rater reliability check, in which three members of the research team coded a random set of responses, and results were compared. The code book was then edited and the check was repeated, resulting in over $92 \%$ agreement across team members.

\section{THEMES IN STUDENT RESPONSES}

We will focus our analysis and discussion on the first three themes identified above - applicability, computational thinking, and usefulness - because responses around these themes offered the most variation; that is to say, they contained four or more sub-themes, which were more complex and informative than the other two themes that were documented. In the discussion at the end of this paper, we will briefly pro- vide more detail on the themes that were excluded from our primary analysis.

For future survey develop, the three core themes presented in the following analysis inform the formation of overarching statement categories. By effectively classifying the subthemes, we are able to craft statements that both accommodate teachers' objectives and resonate with students' opinions within each of these three core themes. Due to the stage we are at in the development of the survey, the following discussion of the core themes is broken down into a presentation of the research findings in the form of the variation in responses within a core theme. The sub-themes within each core theme are described and then we proceed to indicate the implications of these findings for future survey development.

\section{A. Theme 1 - Applicability of Physics Ideas}

Response Variation: The theme of "applicability of physics ideas when coding" (tagged as APP) arose in response to question 5. We documented four different subthemes within the applicability theme, which can be seen in Table I. Physics-specific (PS) applications refer to instances in which the student mentioned specific physics terminology or experiences as evidence that physics ideas are applicable when they code. Students mentioned equations (EQ) or formulae from their physics curricula that they felt applied to their computational task. The learnability (L) and interdisciplinary (ID) sub-themes are similar with one key difference: where some students mentioned that learning to code was easier if they knew their physics ideas, others said that you must know physics in order to code effectively. These four sub-themes together offer significant variance that helps inform the way they should be handled in a future survey.

Survey Development: Student references to equations from physics and math classes is significant. Looking at the APP-EQ example in Table I, the student's response implies a recognition that although equations look different in a programming environment, they are still the same equations from physics class. Future survey statements could incorporate this idea to probe students on whether or not they see this link between the equations in their physics classrooms and in the code. Also, the learnability and interdisciplinary sub-themes emphasize a link between computation and physics, which 
TABLE II. Themes in student responses relating to computational thinking (CT) practices. ${ }^{\text {a }}$

\begin{tabular}{ll}
\hline \hline Sub-themes & Example Responses \\
\hline D - Data management (7/61) & "It's very useful for data to be stored on computers and processed on computers." \\
M - Modeling (11/61) & "It can be used as a model to show how things would move based on the properties of physics." \\
V - Visualization (8/61) & "You are able to show different things being talked about and see a computer version of them." \\
T - Testing/predicting (17/61) & "You might be able to test how things will react on the computer and get a preview." \\
\hline \hline
\end{tabular}

a Numbers in parentheses represent the count of students that mentioned each sub-theme out of the total set of students surveyed.

parallels teachers' intended learning outcomes (e.g., "I want them to be able to use the physics principles in the code."). The student responses for APP-L and APP-ID listed in Table I suggest that by framing questions around the connectedness or disconnectedness or physics and computation, we could reliably evaluate how students feel about the two disciplines in a shared context. The overarching category pertaining to the applicability theme should emphasize the relationship between computation and physics and the translation of physics equations into code. It is important for future survey statements to differentiate student responses that label computation as a tool for learning physics versus physics being necessary for doing computation.

\section{B. Theme 2 - Computational Thinking Practices}

Response Variation: In reviewing the emergent theme of computational thinking (CT) practices, four significant subthemes were discovered (see Table II). Data management (D) refer to when students would talk about the ease of organizing, displaying, and manipulating data. Visualization (V) was heavily emphasized by students who mentioned that seeing the outcome of their work was a big advantage of computation. Multiple students discussed how they could model (M) real-world circumstances with a computer. Finally, testing/predicting $(\mathrm{T})$ was a common sub-theme because many students discussed using computation for trial-and-error scenarios or checking their hypotheses. The prevalence of the visualization sub-theme could suggest that computational activities help students with visualization most of all in terms of computational thinking practices. It could also be a result of them using a programming environment (Glowscript) that is designed to display 3D imagery and simulations. This may not be the case for students who experience coding with strictly scripts and numerical outputs.

Survey Development: When creating a category around computational thinking practices, the sub-themes documented here will serve as guidelines for statement design. Concepts such as data management, modeling, and testing/predicting - the concepts that align most with practices detailed in previous research - merit an array of specific questions to target these sub-themes in the future survey. Computation is a powerful tool for modeling complex physics phenomena (e.g., planetary motion or high speed collisions), and statements about simulating real-world scenarios and building models are especially pertinent. The widespread nature of responses like that displayed for CT-T in Table II, implies that one should incorporate statements involving computer previews and testing hypotheses to see how students feel about computation for experimentation and trial-and-error.

\section{Theme 3 - Usefulness of Computation}

Response Variation: By unpacking the large variety of sub-themes present within usefulness of computation, we gain insight into students' reasoning behind their perceptions of its utility. Table III details the variation of sub-themes that were revealed under the usefulness theme. Many students felt that coding was useful for learning physics, while others felt that computation was useful for doing physics. We felt it was important to separate these ideas into the sub-themes of computation as a learning (L) tool and a physics (P) tool. Some students discussed computing as being useful for accuracy (A) because it offered more speed and precision than working by hand. Others simply stated that it is a useful skill to know for their future $(\mathrm{F})$ or their career because the world is becoming more technological and science is relying more on computation.

Survey Development: Students were relatively split on whether computation was more useful currently for learning and doing physics, or later for their career and future. Statements in the survey should incorporate a time-dependency as discussed in student responses. One should be careful to ensure that the usefulness category does not overlap with other themes; it should adhere strictly to the students' perceived significance of the timeline around computation. Our analysis demonstrated the possibility for double coding across applicability and usefulness themes such as usefulness for helping learning physics (USE-L) and physics ideas apply to computational activities because they help them learn (APP-L). But this shared emphasis on learning when explored did not produce further variation to inform our survey development beyond instilling caution around the possibility for connections across questions exploring usefulness and applicability. 
TABLE III. Themes in student responses relating to usefulness (USE) of computation. ${ }^{\mathrm{a}}$

\begin{tabular}{ll}
\hline \hline Sub-themes & Example Responses \\
\hline L - Learning tool (17/61) & "Computation is useful because it can be used in various branches of science to help us (as students) learn." \\
F - Future/career (16/61) & "Computation is the future of science and the future of the world. An astounding number of jobs are coding \\
& based and this number will only increase. It is best to be prepared for the future." \\
P - Physics tool (8/61) & "It can show science applied. You can use it to run simulations or test physics." \\
A - Accuracy/precision (4/61) & "It can also help data be more specific and closest to the exact numbers as it is computerized." \\
CT - Comp. thinking (35/61) & "It can be used to create programs to demonstrate basic computer science." \\
O - Other (14/61) & Other section refers to unspecified agreements, such as "Yes computation is useful." \\
\hline \hline
\end{tabular}

a Numbers in parentheses represent the count of students that mentioned each sub-theme out of the total set of students surveyed.

\section{DISCUSSION AND CONCLUSIONS}

Our future survey will rely heavily on the creation of statement categories that correspond to the various themes presented in this study including applicability of physics ideas, computational thinking practices, and the usefulness of computation. The associated sub-themes for each theme will govern the creation of statements in the future survey.

An interesting finding was that the sub-themes of computational thinking practices closely align with the practices discussed by Weintrop et al., who give a breakdown of computational thinking consisting of four categories: data practices, modeling and simulating practices, computational problem solving practices, and systems thinking practices [8]. For example, Weintrop and coworkers state, "experts we interviewed frequently referenced the importance of computational thinking with respect to the collection, management, and analysis of data in their work," which is similar to our example in table II, which states "It's very useful for data to be stored on computers and processed on computers." Similar examples can be found in our data that link Weintrop's CT practices to our CT theme. This validates the work of Weintrop et al. in a different context while also indicating that his work will be useful for choosing precise wording around a computational thinking theme [8].

Two other main themes were also evident from student responses: the importance (IMP) of understanding every line of code before using code effectively and the difficulty (DIF) of learning computation. In regard to the IMP theme, the teachers discussed an emphasis on basic concepts as opposed to a comprehensive understanding of programming as a whole. Responses around this theme were somewhat aligned in either "IMP" or "NIMP" (i.e., it is or isn't important to understand every line of code), and this motivated us to leave it out of the primary analysis. As for the "DIF" theme, responses were mostly binary along the lines of "easy" or "difficult." Students occasionally indicated a temporal growth idea of computation being difficult at first but then getting easier over time, but it was infrequent compared to the standard responses, and this was the main motivation for leaving it out of the primary analysis.

Our focus as a whole is to measure student affect and ex- plore the epistemology around computation in high school physics. That being said, the various sub-themes that arose throughout the course of our analysis are not equal with regard to each other or our overarching goals. An example of inequality across themes is that the sub-theme of using computation as a physics tool (USE-P) could possibly be its own category in a larger survey. The accuracy sub-theme of usefulness (USE-A) could be a sub-theme of this category. Certain sub-themes will also be of higher priority when the future survey is developed because they align more with the learning goals of the teachers. The sub-theme of accuracy (USE-A) might prove not to be essential to be included in the final survey as it might not align with teacher learning goals.

It is important to note that the sample size $(N=61)$ is relatively small, and participants in the final questionnaire were ultimately only from one specific classroom with the same instructor. The study would have provided a better portrayal of how students are responding to the questionnaire if a wider spectrum of students (with different classroom experiences) participated. This is a preliminary stage, and in our future work, we intend to utilize more classrooms and a larger spectrum of students.

The themes presented in this work are are influenced by these variables, and a similar study could yield different results according to unique parameters. Overall, we have completed the initial stages of developing an attitudinal survey to evaluate student affect around computation in high school physics. Responses to this initial survey generated themes that will inform the creation of categories for the future survey. The next step in this process will be to present our results to our physics teachers and use their input as a guide for which themes and sub-themes are worth pursuing in a second round of survey data collection.

\section{ACKNOWLEDGMENTS}

We would like to thank the National Science Foundation (Award No. DRL-1741575), the high school teachers for allowing us to collect data in their classes, and the student participants for graciously partaking in our research. 
[1] National Research Council, "A Framework for K-12 Science Education: Practices, Crosscutting Concepts, and Core Ideas," Washington D.C.: The National Academies Press (2012).

[2] E. Behringer and L. Engelheart, "AAPT Recommendations for computational physics in the undergraduate physics curriculum, and the Partnership for Integrating Computation into Undergraduate Physics," Am. J. Phys. 85, 325 (2017).

[3] W.K. Adams, K.K. Perkins, N.S. Podolefsky, M. Dubson, N.D. Finkelstein, and C.E. Wieman, "New instrument for measuring student beliefs about physics and learning physics: The Colorado Learning Attitudes about Science Survey," Phys. Rev. ST Phys. Educ. Res. 2(1), 010101 (2006).

[4] B.M. Zwickl, T. Hirokawa, N. Finkelstein, and H.J. Lewandowski, "Epistemology and expectations survey about experimental physics: Development and initial results," Phys. Rev. ST Phys. Educ. Res. 10(1), 010120 (2014).

[5] I. Halloun and D. Hestenes, "Interpreting VASS Dimensions and Profiles for Physics Students," Sci. and Educ. 7(6), 553 (1998).
[6] E.F. Redish, J.M. Saul, and R.N. Steinberg, "Student Expectations in Introductory Physics,” Am. J. Phys. 66(3), 212 (1998).

[7] J. Barbera, W.K. Adams, C.E. Wieman, and K.K. Perkins, "Modifying and Validating the Colorado Learning Attitudes about Science Survey for Use in Chemistry," J. Chem. Educ. 85(10), 1435 (2008).

[8] D. Weintrop, E. Beheshti, M. Horn, K. Orton, K. Jona, L. Trouille, and U. Wilensky, "Defining Computational Thinking for Mathematics and Science Classrooms," J. Sci. Educ. Tech. 25(1), 127 (2016).

[9] V.K. Otero and D.B. Harlow, "Getting Started in Qualitative Physics Education Research,” Rev. PER 2(31) (2009)

[10] R.E. Boyatzis, "Transforming qualitative information: Thematic analysis and code development," Thousand Oaks: Sage Publications (1998).

[11] T.E. Bott, "Questionnaire Development," External Document on GoogleDocs (2019). 